\title{
Streamlines in the Two-Dimensional Spreading of a Thin Fluid Film: Blowing and Suction Velocity Proportional to the Spatial Gradient of the Height
}

\author{
N. Modhien1, D. P. Mason', E. Momoniat',3 \\ ${ }^{1}$ DSI-NRF Centre of Excellence in Mathematical and Statistical Sciences, School of Computer Science and Applied Mathematics, \\ University of the Witwatersrand, Johannesburg, South Africa \\ ${ }^{2}$ Data Science across Disciplines Research Group, Institute for the Future of Knowledge, University of Johannesburg, \\ Johannesburg, South Africa \\ ${ }^{3}$ Department of Mathematics and Applied Mathematics, University of Johannesburg, Johannesburg, South Africa \\ Email: Naeemah.Modhien@wits.ac.za
}

How to cite this paper: Modhien, N., Mason, D.P. and Momoniat, E. (2021) Streamlines in the Two-Dimensional Spreading of a Thin Fluid Film: Blowing and Suction Velocity Proportional to the Spatial Gradient of the Height. Journal of Applied Mathematics and Physics, 9, 2733-2756.

https://doi.org/10.4236/jamp.2021.911176

Received: September 26, 2021

Accepted: November 12, 2021

Published: November 15, 2021

Copyright $\odot 2021$ by author(s) and Scientific Research Publishing Inc. This work is licensed under the Creative Commons Attribution International License (CC BY 4.0).

http://creativecommons.org/licenses/by/4.0/

cc) (i) Open Access

\begin{abstract}
The aim of this investigation is to determine the effect of fluid leak-off (suction) and fluid injection (blowing) at the horizontal base on the two-dimensional spreading under the gravity of a thin film of viscous incompressible fluid by studying the evolution of the streamlines in the thin film. It is assumed that the normal component of the fluid velocity at the base is proportional to the spatial gradient of the height of the film. Lie symmetry methods for partial differential equations are applied. The invariant solution for the surface profile is derived. It is found that the thin fluid film approximation is satisfied for weak to moderate leak-off and for the whole range of fluid injection. The streamlines are derived and plotted by solving a cubic equation numerically. For fluid injection, there is a dividing streamline originating at the stagnation point at the base which separates the flow into two regions, a lower region consisting mainly of rising fluid and an upper region consisting mainly of descending fluid. An approximate analytical solution for the dividing streamline is derived. It generates an approximate $\mathrm{V}$-shaped surface along the length of the two-dimensional film with the vertex of each section the stagnation point. It is concluded that the fluid flow inside the thin film can be visualised by plotting the streamlines. Other models relating the fluid velocity at the base to the height of the thin film can be expected to contain a dividing streamline originating at a stagnation point and dividing the flow into a lower region
\end{abstract}


of rising fluid and an upper region of descending fluid.

\section{Keywords}

Thin Fluid Film, Suction and Blowing, Invariant Solution, Streamlines, Dividing Streamline

\section{Introduction}

In a recent paper [1] we investigated the effect of fluid leak-off (suction) and fluid injection (blowing) at the horizontal base on the two-dimensional spreading under the gravity of a thin film of viscous incompressible fluid. We derived and analysed the plots of the streamlines to understand the fluid flow in the thin film on which the evolution of the surface profile depended. The system of equations was closed by making the assumption that the normal component of the fluid velocity at the base, $V_{n}$, is proportional to the height of the thin film at that point. With this assumption, an analytical solution for the surface profile could be derived and the streamlines were plotted by solving a cubic equation numerically. In this model, the magnitude of the suction and blowing is greatest near the centre line of the thin film because the height is greatest on the centre line and is least near the moving contact lines where the height vanishes.

In this paper, we will assume that $V_{n}$ is proportional to the spatial gradient of the height. An analytical solution for the surface profile can again be derived. We will plot the streamlines and investigate their properties and compare them with the results obtained in [1]. In this model, the magnitude of the suction and blowing is least near the centre line on which the spatial gradient vanishes and is greatest near the moving contact lines on which the magnitude of the spatial gradient is infinite.

Investigations of the evolution of a thin fluid film with a free surface generally concentrate on the surface profile [2] [3] [4]. In this paper, we investigate the properties and evolution of the thin film by deriving and analysing the streamlines of the fluid flow in the thin film.

Mason and Momoniat [5] made the assumption that $V_{n}$ is proportional to the spatial gradient of the height in the axisymmetric spreading under the gravity of a thin liquid drop with suction and blowing at the base. They did not investigate the streamlines in the liquid drop. We will use Lie group analysis of differential equations to reduce the nonlinear diffusion equation to an ordinary differential equation and derive an analytical solution. This is a powerful and systematic method that has been applied successfully in other investigations in thin fluid film theory. It can be used to analyse the evolution of a thin fluid film from a point or line source of fluid and from a fluid film with an initial non-zero half-width as considered here. A literature review of the relevant papers of thin fluid film theory and of the application of Lie group analysis to thin fluid films was given in [1].

An outline of the paper is as follows. In Section 2 a summary is given of the 
results needed from [1]. In Section 3 the invariant solution is derived from the Lie group analysis performed in [1]. In Section 4 the evolution with time of the fluid variables is analysed. In Section 5 the streamlines are derived and plotted and in Section 6 an approximate analytical solution for the dividing streamline is obtained. Finally, the conclusions are summarised in Section 7.

A nomenclature is presented in Table 1.

\section{Summary of Results}

The fluid is viscous and incompressible. The spreading of the thin fluid film is two-dimensional as shown in Figure 1. There is suction (fluid leak-off) or blowing (fluid injection) at the base. The moving contact lines are $x= \pm w(t)$ and the height of the thin fluid film is $z=h(t, x)$. The fluid film is infinite in the $y$-direction. The variables are dimensionless.

The thin fluid film approximation is imposed. The $x$ - and $z$-components of the fluid velocity satisfy

$$
\begin{gathered}
v_{x}(t, x, z)=\left(\frac{1}{2} z^{2}-z h(t, x)\right) \frac{\partial h}{\partial x}(t, x), \\
v_{z}(t, x, z)=-\frac{1}{6} z^{3} \frac{\partial^{2} h}{\partial x^{2}}(t, x)+\frac{1}{4} z^{2} \frac{\partial^{2}}{\partial x^{2}}\left(h^{2}(t, x)\right)+v_{n}(t, x) .
\end{gathered}
$$

Table 1. Nomenclature for significant variables and parameters.

\begin{tabular}{ll}
\hline & \multicolumn{1}{c}{ Nomenclature } \\
\hline$v_{n}(t, x)$ & fluid injection/leak-off velocity \\
$h(t, x)$ & height of thin film \\
$w(t)$ & half-width of thin fluid film \\
$\eta$ & $x / w(t)$ scaled similarity variable \\
$f(\eta)$ & invariant solution for $h(t, x)$ \\
$g(\eta)$ & invariant solution for $v_{n}(t, x)$ \\
$\psi(t, x, z)$ & stream function \\
$\alpha$ & $c_{4} / c_{2} \quad$ where $c_{4}$ and $c_{2}$ are constants in the Lie point symmetry \\
$\beta^{*}$ & constant in equation $g(\eta)=-\beta^{*} \eta \frac{\partial f}{\partial \eta}$ \\
$\beta$ & $\begin{array}{l}2 \beta^{*} / 5\left(1-\beta^{*}\right) \\
\varepsilon(t)\end{array}$ \\
$\begin{array}{l}h(t, 0) / w(t) \text { thin fluid film ratio } \\
z^{*}\end{array}$ & $\begin{array}{l}\text { point of intersection of dividing streamline with free surface in } \\
\left(\eta, z^{*}\right) \text {-plane }\end{array}$ \\
$\left(\eta_{P}, z_{P}^{*}\right)$ & $\begin{array}{l}\text { angle dividing streamline makes with positive } x \text {-axis } \\
(x, z) \text {-plane }\end{array}$ \\
\hline &
\end{tabular}




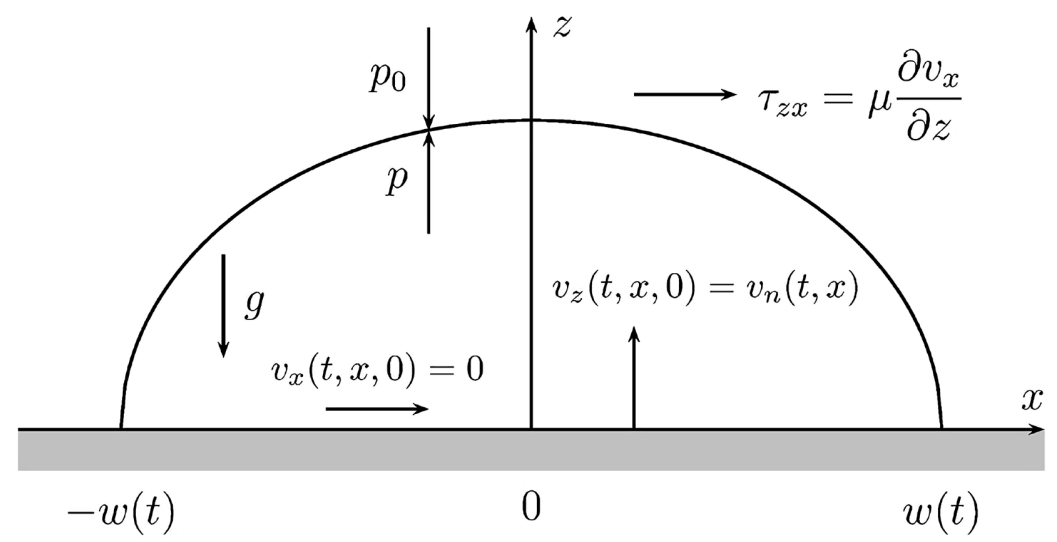

Figure 1. Spreading of a two-dimensional thin fluid film under gravity illustrating the coordinate system, body force and boundary conditions.

The height $h(t, x)$ satisfies the nonlinear diffusion equation

$$
\frac{\partial h}{\partial t}=\frac{1}{3} \frac{\partial}{\partial x}\left(h^{3} \frac{\partial h}{\partial x}\right)+v_{n}(t, x)
$$

where $v_{n}(t, x)$ is the normal component of the fluid velocity at the base. The boundary conditions are that at the moving contact lines the height vanishes,

$$
h(t, \pm w(t))=0
$$

and the fluid flux vanishes,

$$
h^{3}(t, \pm w(t)) \frac{\partial h}{\partial x}(t, \pm w(t))=0 .
$$

The balance law for fluid volume is

$$
\frac{\mathrm{d} V}{\mathrm{~d} t}=2 \int_{0}^{w(t)} v_{n}(t, x) \mathrm{d} x
$$

where $V(t)$ is the total volume of the thin film per unit length in the $y$-direction:

$$
V(t)=2 \int_{0}^{w(t)} h(t, x) \mathrm{d} x .
$$

An invariant solution is derived using Lie group analysis for differential equations [1] [6]. The nonlinear diffusion Equation (2.3) admits the Lie point symmetry

$$
X=\left(c_{1}+c_{2} t\right) \frac{\partial}{\partial t}+\left(c_{3}+c_{4} x\right) \frac{\partial}{\partial x}+\frac{1}{3}\left(2 c_{4}-c_{2}\right) h \frac{\partial}{\partial h}
$$

provided $v_{n}(t, x)$ satisfies

$$
\left(c_{1}+c_{2} t\right) \frac{\partial v_{n}}{\partial t}+\left(c_{3}+c_{4} x\right) \frac{\partial v_{n}}{\partial x}=\frac{2}{3}\left(c_{4}-2 c_{2}\right) v_{n} .
$$

Using the Lie point symmetry (2.8) the nonlinear diffusion Equation (2.3) is reduced to an ordinary differential equation. There are two cases, the general case, $c_{2} \neq 0$ and the special case, $c_{2}=0$.

When $c_{2} \neq 0$ the invariant solution is obtained by solving the ordinary dif- 
ferential equation

$$
\frac{\mathrm{d}}{\mathrm{d} \eta}\left(f^{3} \frac{\mathrm{d} f}{\mathrm{~d} \eta}\right)+3 \frac{\mathrm{d}}{\mathrm{d} \eta}(\eta f)+\left(\frac{1}{\alpha}-5\right) f+3 g=0,
$$

where

$$
\alpha=\frac{c_{4}}{c_{2}}, \quad \eta=\frac{x}{w(t)},
$$

subject to the boundary conditions

$$
\begin{gathered}
f( \pm 1)=0, \\
f^{3}( \pm 1) \frac{\mathrm{d}}{\mathrm{d} \eta} f( \pm 1)=0
\end{gathered}
$$

and to the balance law for fluid volume,

$$
\frac{1}{3}\left(5-\frac{1}{\alpha}\right) \int_{0}^{1} f(\eta) \mathrm{d} \eta=\int_{0}^{1} g(\eta) \mathrm{d} \eta .
$$

The invariant solutions for the fluid variables are

$$
\begin{gathered}
h(t, x)=\left(1+\frac{t}{\alpha f^{3}(0)}\right)^{\frac{2}{3}\left(\alpha-\frac{1}{2}\right)} \frac{f(\eta)}{f(0)}, \\
v_{n}(t, x)=\frac{1}{f^{4}(0)}\left(1+\frac{t}{\alpha f^{3}(0)}\right)^{\frac{2}{3}(\alpha-2)} g(\eta), \\
w(t)=\left(1+\frac{t}{\alpha f^{3}(0)}\right)^{\alpha}, \\
V(t)=V_{0}\left(1+\frac{t}{\alpha f^{3}(0)}\right)^{\frac{5}{3}\left(\alpha-\frac{1}{5}\right)},
\end{gathered}
$$

where

$$
V_{0}=2 \int_{0}^{1} \frac{f(\eta)}{f(0)} \mathrm{d} \eta .
$$

The Lie point symmetry which generates the invariant solution is

$$
X=\left(f^{3}(0)+\frac{1}{\alpha} t\right) \frac{\partial}{\partial t}+x \frac{\partial}{\partial x}+\frac{1}{3}\left(2-\frac{1}{\alpha}\right) h \frac{\partial}{\partial h} .
$$

when $c_{2}=0$ the invariant solution is obtained by solving the ordinary differential equation

$$
\frac{\mathrm{d}}{\mathrm{d} \eta}\left(f^{3} \frac{\mathrm{d} f}{\mathrm{~d} \eta}\right)+3 \frac{\mathrm{d}}{\mathrm{d} \eta}(\eta f)-5 f+3 g=0
$$

subject to the boundary conditions (2.12) and (2.13) and to the balance law for fluid volume

$$
\frac{5}{3} \int_{0}^{1} f(\eta) \mathrm{d} \eta=\int_{0}^{1} g(\eta) \mathrm{d} \eta .
$$


The invariant solution is of the form

$$
\begin{gathered}
h(t, x)=\exp \left(\frac{2}{3} \frac{t}{f^{3}(0)}\right) \frac{f(\eta)}{f(0)}, \\
v_{n}(t, x)=\frac{1}{f^{4}(0)} \exp \left(\frac{2}{3} \frac{t}{f^{3}(0)}\right) g(\eta), \\
w(t)=\exp \left(\frac{t}{f^{3}(0)}\right), \\
V(t)=V_{0} \exp \left(\frac{5}{3} \frac{t}{f^{3}(0)}\right),
\end{gathered}
$$

where $V_{0}$ is given by (2.19) and $\eta$ is given by (2.11). The Lie point symmetry which generates the invariant solution is

$$
X=f^{3}(0) \frac{\partial}{\partial t}+x \frac{\partial}{\partial x}+\frac{2}{3} h \frac{\partial}{\partial h} .
$$

Unlike (2.20), the Lie point symmetry (2.27) is not a scaling symmetry. It can be verified that the system of equations (2.21) to (2.26) for $c_{2}=0$ can be obtained from the Equations (2.10) to (2.20) for $c_{2} \neq 0$ by letting $\alpha \rightarrow \infty$.

For the two-dimensional flow of an incompressible fluid the streamlines can be obtained from the stream function. The stream function $\psi(t, x, z)$ satisfies

$$
v_{x}(t, x, z)=\frac{\partial \psi}{\partial z}, \quad v_{z}(t, x, z)=-\frac{\partial \psi}{\partial x}
$$

and the streamlines at time $t$ are the curves

$$
\psi(t, x, z)=k(t)
$$

where $k(t)$ is given a range of discrete values. In the $(x, z)$-plane the streamlines at time $t$ are obtained by solving numerically for $z$ as a function of $x$ the cubic equation [1]

$$
A(t, x) z^{3}+B(t, x) z^{2}+C(t, x)=K(t)
$$

for a range of discrete values of $K(t)$ where

$$
\begin{aligned}
& A(t, x)=\frac{1}{6} \frac{\partial h}{\partial x}(t, x), \\
& B(t, x)=-\frac{1}{2} h(t, x) \frac{\partial h}{\partial x}, \\
& C(t, x)=-\int_{0}^{x} v_{n}(t, s) \mathrm{d} s .
\end{aligned}
$$

We will also consider the streamlines in the $\left(\eta, z^{*}\right)$-plane where $\eta=x / w(t)$ and $z^{*}=z / h(t, 0)$. In the $\left(\eta, z^{*}\right)$-plane the streamlines satisfy the cubic equation [1]

$$
A^{*}(x, \eta) z^{* 3}+B^{*}(t, \eta) z^{* 2}+C^{*}(t, \eta)=K(t)
$$

where 


$$
\begin{aligned}
& A^{*}(t, \eta)=h^{3}(t, 0) A(t, x), \\
& B^{*}(t, \eta)=h^{2}(t, 0) B(t, x), \\
& C^{*}(t, \eta)=C(t, x) .
\end{aligned}
$$

In order to close the systems of equations and complete the formulation of the problem an assumption needs to be made on $v_{n}(t, x)$. In [1] it was assumed that $v_{n}(t, x)$ is proportional to $h(t, x)$. In this paper it will be assumed that $v_{n}(t, x)$ is proportional to the spatial gradient $x \frac{\partial h}{\partial x}(t, x)$.

\section{Invariant Solution}

We first consider the general case $c_{2} \neq 0$. Assume that

$$
g(\eta)=-\beta^{*} \eta \frac{\mathrm{d} f}{\mathrm{~d} \eta}
$$

where $-\infty<\beta^{*}<\infty$ is a constant. The balance law (2.14) becomes

$$
\frac{1}{3}\left(5-\frac{1}{\alpha}\right) \int_{0}^{1} f(\eta) \mathrm{d} \eta=-\beta^{*} \int_{0}^{1} \eta \frac{\mathrm{d} f}{\mathrm{~d} \eta} \mathrm{d} \eta
$$

and by integrating by parts we find that

$$
\left[\beta^{*}-\frac{1}{3}\left(5-\frac{1}{\alpha}\right)\right] \int_{0}^{1} f(\eta) \mathrm{d} \eta=0 .
$$

Hence

$$
\alpha=\frac{1}{5-3 \beta^{*}}, \quad \beta^{*} \neq \frac{5}{3} .
$$

The differential Equation (2.10) with $\alpha$ given by (3.4) becomes

$$
\frac{\mathrm{d}}{\mathrm{d} \eta}\left(f^{3} \frac{\mathrm{d} f}{\mathrm{~d} \eta}\right)+3\left(1-\beta^{*}\right) \frac{\mathrm{d}}{\mathrm{d} \eta}(\eta f)=0
$$

which can now be integrated subject to the boundary conditions (2.12) and (2.13). We obtain

$$
f^{3}(\eta)=\frac{9}{2}\left(1-\beta^{*}\right)\left(1-\eta^{2}\right), \quad-1 \leq \eta \leq 1
$$

and therefore

$$
f(\eta)=f(0)\left(1-\eta^{2}\right)^{\frac{1}{3}}, \quad f(0)=\left[\frac{9}{2}\left(1-\beta^{*}\right)\right]^{\frac{1}{3}} .
$$

For $\beta^{*}>1$ there is one real solution for $f(0)$ which is negative and two complex conjugate solutions which we do not consider. From (2.15) to (2.18) we see that the real negative solution gives a positive solution for $h(t, x)$ and that the other fluid variables depend only on $f^{3}(0)$. Hence $-\infty<\beta^{*}<\infty$ with $\beta^{*} \neq \frac{5}{3}$.

In order to determine the physical significance of $\beta^{*}$ consider $v_{n}(t, x)$. From (2.16), 


$$
\left.v_{n}(t, x)=\frac{4 \beta^{*}}{27\left(1-\beta^{*}\right)}\left[1+\frac{2\left(5-3 \beta^{*}\right)}{9\left(1-\beta^{*}\right)}\right]^{2\left(2 \beta^{*}-3\right)}\right]^{5-3 \beta^{*}} \frac{\eta^{2}}{\left(1-\eta^{2}\right)^{2 / 3}} .
$$

We introduce the new parameter $\beta$ defined by

$$
\beta=\frac{2}{5} \frac{\beta^{*}}{1-\beta^{*}} \text {. }
$$

The factor $\frac{2}{5}$ is a normalisation factor to give $\beta=-1$ for the special case $\beta^{*}=\frac{5}{3}$. The range of $\beta$ is $-\infty<\beta<\infty$. Equation (3.8) becomes

$$
v_{n}(t, x)=\frac{10}{27} \beta\left[1+\frac{10}{9}(1+\beta) t\right]^{-\frac{6+5 \beta}{5(1+\beta)}} \frac{\eta^{2}}{\left(1-\eta^{2}\right)^{2 / 3}} .
$$

The normal fluid velocity at the base has the same sign as $\beta$ and $\beta>0$ describes blowing (fluid injection) into the thin film, $\beta=0$ describes an impermeable base and $\beta<0$ describes suction (fluid leak-off) at the base. The other fluid variables are

$$
\begin{gathered}
w(t)=\left[1+\frac{10}{9}(1+\beta) t\right]^{\frac{2+5 \beta}{1(1+\beta)}}, \\
V(t)=V_{0}\left[1+\frac{10}{9}(1+\beta) t\right]^{\frac{\beta}{2(1+\beta)}}, \\
V_{0}=2 \int_{0}^{1} \eta\left(1-\eta^{2}\right)^{\frac{1}{3}} \mathrm{~d} \eta=\frac{3}{4}, \\
h(t, x)=\left[1+\frac{10}{9}(1+\beta) t\right]^{-\frac{1}{5(1+\beta)}}\left(1-\eta^{2}\right)^{\frac{1}{3}} .
\end{gathered}
$$

From (3.10), (3.11) and (3.14)

$$
v_{n}(t, x)=-\frac{5}{9} \beta w(t)^{-\frac{3(4+5 \beta)}{2+5 \beta}} x \frac{\partial h}{\partial x} .
$$

We see that the physical significance of the solution is that $v_{n}(t, x)$ is proportional to $-x \frac{\partial h}{\partial x}$ which like $v_{n}(t, x)$ is an even function of $x$.

Consider now the special case, $c_{2}=0$. Assuming (3.1), the balance law (2.22) becomes

$$
\left(\frac{5}{3}-\beta^{*}\right) \int_{0}^{1} f(\eta) \mathrm{d} \eta=0
$$

and therefore $\beta^{*}=\frac{5}{3}$ and $\beta=-1$ The differential Equation (2.21) becomes

$$
\frac{\mathrm{d}}{\mathrm{d} \eta}\left(f^{3} \frac{\mathrm{d} f}{\mathrm{~d} \eta}\right)-2 \frac{\mathrm{d}}{\mathrm{d} \eta}(\eta f)=0 .
$$


Integrating and imposing the boundary conditions we obtain

$$
f(\eta)=f(0)\left(1-\eta^{2}\right)^{\frac{1}{3}}, \quad f(0)=-3^{\frac{1}{3}} .
$$

Hence from (2.23) to (2.26),

$$
\begin{gathered}
h(t, x)=\exp \left(-\frac{2}{9} t\right)\left(1-\eta^{2}\right)^{\frac{1}{3}}, \\
v_{n}(t, x)=-\frac{10}{27} \exp \left(-\frac{2}{9} t\right) \frac{\eta^{2}}{\left(1-\eta^{2}\right)^{2 / 3}}, \\
w(t)=\exp \left(-\frac{1}{3} t\right), \\
V(t)=V_{0} \exp \left(-\frac{5}{9} t\right)
\end{gathered}
$$

where $V_{0}$ is given by (3.13).

\section{Analysis of the Solution}

We will determine the evolution in time of the fluid variables, especially $w(t)$ and $h(t, 0)$ and investigate the fluid velocity on the centre line, $x=0$.

\subsection{Time Evolution of the Fluid Variables}

Consider first the thin fluid film approximation [1]. From (3.11) and (3.14) for $\beta \neq-1$,

$$
\varepsilon(t)=\frac{h(t, 0)}{w(t)}=\left[1+\frac{10}{9}(1+\beta) t\right]^{-\frac{4+5 \beta}{10(1+\beta)}}
$$

and from (3.19) and (3.21) for $\beta=-1$,

$$
\varepsilon(t)=\frac{h(t, 0)}{w(t)}=\exp \left(\frac{1}{9} t\right) .
$$

For $-\infty<\beta<-1, \varepsilon(t) \rightarrow \infty$ as $t \rightarrow t_{2}$ where

$$
t_{2}=-\frac{9}{10(1+\beta)}>0 \text {, }
$$

for $\beta=-1, \varepsilon(t) \rightarrow \infty$ exponentially as $t \rightarrow \infty$ while for $-1<\beta<-\frac{4}{5}$,

$\varepsilon(t) \rightarrow \infty$ algebraically as $t \rightarrow \infty$. For $\beta=-\frac{4}{5}, \varepsilon(t)=1$ and therefore remains constant and for $-\frac{4}{5}<\beta<\infty, \varepsilon(t) \rightarrow 0$ as $t \rightarrow \infty$. Since it is assumed that the thin fluid film approximation is satisfied initially it will remain satisfied for all time for $-\frac{4}{5} \leq \beta \leq \infty$ which is the range of $\beta$ for which the solution is valid physically. The special solution for $\beta=-1$ with exponential behaviour and the solution in the range $-\infty<\beta<-1$ in which the limit is attained in fi- 
nite time lie outside the physical range. Interestingly the thin film approximation is satisfied for the whole range of blowing, $0<\beta \leq \infty$, which also has the most interesting streamlines. The spreading due to gravity is the dominant mechanism and is stronger than the effect of blowing on the height. The results are summarised in Table 2.

Consider next the half-width of the base, $w(t)$, for the range $-\frac{4}{5} \leq \beta \leq \infty$ in which the solution applies. For this range, $w(t)$ is given by (3.11). For $-\frac{4}{5} \leq \beta<\frac{2}{5}, w(t) \rightarrow 0$ as $t \rightarrow \infty$ but in such a way that the thin fluid film approximation remains valid. The two-dimensional thin film shrinks to a line as $t \rightarrow \infty$ due to suction. For $\beta=\frac{2}{5}, w(t)$ remains constant as the fluid evolves and for $-\frac{2}{5}<\beta \leq \infty, w(t) \rightarrow \infty$ as $t \rightarrow \infty$. The behaviour of $w(t)$ for the full range, $-\infty \leq \beta \leq \infty$ is presented in Table 2 .

Consider next $h(t, 0)$ for the physical range, $-\frac{4}{5} \leq \beta \leq \infty$. From (3.14)

Table 2. Time evolution of $\varepsilon(t), w(t), h(t, 0)$ and $V(t)$ for $-\infty \leq \beta \leq \infty$. The finite time $t_{2}$ is defined by (4.3). The thin fluid film approximation is satisfied for all time only for $-\frac{4}{5} \leq \beta \leq \infty$.

\begin{tabular}{|c|c|c|c|c|c|c|c|c|}
\hline$\beta$ & $\varepsilon(t)$ & $\frac{\mathrm{d} \varepsilon}{\mathrm{d} t}$ & $w(t)$ & $\frac{\mathrm{d} w}{\mathrm{~d} t}$ & $h(t, 0)$ & $\frac{\mathrm{d} h}{\mathrm{~d} t}(t, 0)$ & $V(t)$ & $\frac{\mathrm{d} V}{\mathrm{~d} t}$ \\
\hline$-\infty \leq \beta<-1$ & $\begin{array}{c}\varepsilon(t) \rightarrow \infty \\
t \rightarrow t_{2}\end{array}$ & $>0$ & $\begin{array}{c}w(t) \rightarrow 0 \\
t \rightarrow t_{2}\end{array}$ & $<0$ & $\begin{array}{c}h(t, 0) \rightarrow 0 \\
t \rightarrow t_{2}\end{array}$ & $<0$ & $\begin{array}{c}V(t) \rightarrow 0 \\
t \rightarrow t_{2}\end{array}$ & $<0$ \\
\hline$\beta=-1$ & $\begin{array}{c}\varepsilon(t) \rightarrow \infty \\
t \rightarrow \infty \\
\text { Exponentially }\end{array}$ & $>0$ & $\begin{array}{c}w(t) \rightarrow 0 \\
t \rightarrow \infty \\
\text { Exponentially }\end{array}$ & $<0$ & $\begin{array}{c}h(t, 0) \rightarrow 0 \\
\quad t \rightarrow \infty \\
\text { Exponentially }\end{array}$ & $<0$ & $\begin{array}{c}V(t) \rightarrow 0 \\
t \rightarrow \infty \\
\text { Exponentially }\end{array}$ & $<0$ \\
\hline$-1<\beta<-\frac{4}{5}$ & $\begin{array}{c}\varepsilon(t) \rightarrow \infty \\
t \rightarrow \infty\end{array}$ & $>0$ & $\begin{array}{c}w(t) \rightarrow 0 \\
t \rightarrow \infty\end{array}$ & $<0$ & $\begin{array}{c}h(t, 0) \rightarrow 0 \\
t \rightarrow \infty\end{array}$ & $<0$ & $\begin{array}{c}V(t) \rightarrow 0 \\
t \rightarrow \infty\end{array}$ & $<0$ \\
\hline$\beta=-\frac{4}{5}$ & $\varepsilon(t)=1$ & 0 & $\begin{array}{c}w(t) \rightarrow 0 \\
t \rightarrow \infty\end{array}$ & $<0$ & $\begin{array}{c}h(t, 0) \rightarrow 0 \\
t \rightarrow \infty\end{array}$ & $<0$ & $\begin{array}{c}V(t) \rightarrow 0 \\
t \rightarrow \infty\end{array}$ & $<0$ \\
\hline$-\frac{4}{5}<\beta<-\frac{2}{5}$ & $\begin{array}{c}\varepsilon(t) \rightarrow 0 \\
t \rightarrow \infty\end{array}$ & $<0$ & $\begin{array}{c}w(t) \rightarrow 0 \\
t \rightarrow \infty\end{array}$ & $<0$ & $\begin{array}{c}h(t, 0) \rightarrow 0 \\
t \rightarrow \infty\end{array}$ & $<0$ & $\begin{array}{c}V(t) \rightarrow 0 \\
t \rightarrow \infty\end{array}$ & $<0$ \\
\hline$\beta=-\frac{2}{5}$ & $\begin{array}{c}\varepsilon(t) \rightarrow 0 \\
t \rightarrow \infty\end{array}$ & $<0$ & $w(t)=1$ & 0 & $\begin{array}{c}h(t, 0) \rightarrow 0 \\
t \rightarrow \infty\end{array}$ & $<0$ & $\begin{array}{c}V(t) \rightarrow 0 \\
t \rightarrow \infty\end{array}$ & $<0$ \\
\hline$-\frac{2}{5}<\beta<0$ & $\begin{array}{c}\varepsilon(t) \rightarrow 0 \\
t \rightarrow \infty\end{array}$ & $<0$ & $\begin{array}{c}w(t) \rightarrow \infty \\
t \rightarrow \infty\end{array}$ & $>0$ & $\begin{array}{c}h(t, 0) \rightarrow 0 \\
t \rightarrow \infty\end{array}$ & $<0$ & $\begin{array}{c}V(t) \rightarrow 0 \\
t \rightarrow \infty\end{array}$ & $<0$ \\
\hline$\beta=0$ & $\begin{array}{c}\varepsilon(t) \rightarrow 0 \\
t \rightarrow \infty\end{array}$ & $<0$ & $\begin{array}{c}w(t) \rightarrow \infty \\
t \rightarrow \infty\end{array}$ & $>0$ & $\begin{array}{c}h(t, 0) \rightarrow 0 \\
t \rightarrow \infty\end{array}$ & $<0$ & $V=\frac{3}{4}$ & 0 \\
\hline $0<\beta \leq \infty$ & $\begin{array}{c}\varepsilon(t) \rightarrow 0 \\
t \rightarrow \infty\end{array}$ & $<0$ & $\begin{array}{c}w(t) \rightarrow \infty \\
t \rightarrow \infty\end{array}$ & $>0$ & $\begin{array}{c}h(t, 0) \rightarrow 0 \\
t \rightarrow \infty\end{array}$ & $<0$ & $\begin{array}{c}V(t) \rightarrow \infty \\
t \rightarrow \infty\end{array}$ & $>0$ \\
\hline
\end{tabular}




$$
h(t, 0)=\left[1+\frac{10}{9}(1+\beta) t\right]^{-\frac{1}{5(1+\beta)}}
$$

and

$$
\frac{\mathrm{d} h}{\mathrm{~d} t}(t, 0)=v_{z}(t, 0, h)=-\frac{2}{9}\left[1+\frac{10}{9}(1+\beta) t\right]^{-\frac{6+5 \beta}{5(1+\beta)}}
$$

We see that $\frac{\mathrm{d} h}{\mathrm{~d} t}(t, 0)<0$ for $-\frac{4}{5} \leq \beta \leq \infty$ and that $h(t, 0) \rightarrow 0$ as $t \rightarrow \infty$, even for blowing when $0<\beta \leq \infty$. The behaviour of $h(t, 0)$ as $t$ evolves for the full range, $-\infty<\beta<\infty$, is given in Table 2 .

The evolution of $V(t)$ is summarised in Table 2. The time evolution of $v_{n}(t, x)$ for a fixed value of $\eta$ in the range $0<\eta<1$ is given in Table 3. Also given in Table 3 is the time evolution of $v_{z}(t, 0, h)$. We see from (4.5) and (3.10) that $v_{n}(t, x)$ and $v_{z}(t, 0, h)$ evolve with time in the same way.

Table 3. Time evolution of $v_{n}(t, x)$ for $x \neq 0$ and of $v_{z}(t, 0, h)=\frac{\mathrm{d} h}{\mathrm{~d} t}(t, 0)$ for $-\infty \leq \beta \leq \infty$. The finite time $t_{2}$ is defined by Equation (4.3). The thin fluid film ap-

\begin{tabular}{|c|c|c|c|}
\hline$\beta$ & $v_{n}(t, x)$ & $\frac{\partial}{\partial t} v_{n}(t, x)$ & $v_{z}(t, 0, h)$ \\
\hline$-\infty \leq \beta<-\frac{6}{5}$ & $\begin{array}{c}v_{n}(t, x) \rightarrow-\infty \\
t \rightarrow t_{2}\end{array}$ & $\frac{\partial v_{n}}{\partial t}<0$ & $\begin{array}{c}v_{z}(t, 0, h) \rightarrow-\infty \\
t \rightarrow t_{2}\end{array}$ \\
\hline$\beta=-\frac{6}{5}$ & $v_{n}(t, x)=-\frac{4}{9} \frac{\eta^{2}}{1-\eta^{2}}$ & $\frac{\partial v_{n}}{\partial t}=0$ & $v_{z}(t, 0, h)=-\frac{2}{9}$ \\
\hline$-\frac{6}{5}<\beta<-1$ & $\begin{array}{c}v_{n}(t, x) \rightarrow 0 \\
t \rightarrow t_{2}\end{array}$ & $\frac{\partial v_{n}}{\partial t}>0$ & $\begin{array}{c}v_{z}(t, 0, h) \rightarrow 0 \\
t \rightarrow t_{2}\end{array}$ \\
\hline$\beta=-1$ & $\begin{array}{c}v_{n}(t, x) \rightarrow 0 \\
\quad t \rightarrow \infty \\
\text { Exponentially }\end{array}$ & $\frac{\partial v_{n}}{\partial t}>0$ & $\begin{array}{c}v_{z}(t, 0, h) \rightarrow 0 \\
\quad t \rightarrow \infty \\
\text { Exponentially }\end{array}$ \\
\hline$-1<\beta<-\frac{4}{5}$ & $\begin{array}{c}v_{n}(t, x) \rightarrow 0 \\
t \rightarrow \infty\end{array}$ & $\frac{\partial v_{n}}{\partial t}>0$ & $\begin{array}{c}v_{z}(t, 0, h) \rightarrow 0 \\
t \rightarrow \infty\end{array}$ \\
\hline$-\frac{4}{5} \leq \beta<0$ & $\begin{array}{c}v_{n}(t, x) \rightarrow 0 \\
t \rightarrow \infty\end{array}$ & $\frac{\partial v_{n}}{\partial t}>0$ & $\begin{array}{c}v_{z}(t, 0, h) \rightarrow 0 \\
t \rightarrow \infty\end{array}$ \\
\hline$\beta=0$ & $v_{n}(t, x)=0$ & $\frac{\partial v_{n}}{\partial t}=0$ & $\begin{array}{c}v_{z}(t, 0, h) \rightarrow 0 \\
t \rightarrow \infty\end{array}$ \\
\hline $0<\beta \leq \infty$ & $\begin{array}{c}v_{n}(t, x) \rightarrow 0 \\
t \rightarrow \infty\end{array}$ & $\frac{\partial v_{n}}{\partial t}<0$ & $\begin{array}{c}v_{z}(t, 0, h) \rightarrow 0 \\
t \rightarrow \infty\end{array}$ \\
\hline
\end{tabular}
proximation is satisfied for all time only for $-\frac{4}{5} \leq \beta \leq \infty$. 


\subsection{Fluid Velocity on Centre Line}

On the centre line, $x=0$, we have $v_{x}(t, 0, z)=0$ by (2.1). From (2.2), since $v_{n}(t, 0)=0$ by (3.10) and (3.20),

$$
v_{z}(t, 0, z)=-\left.\frac{1}{6} \frac{\partial^{2} h}{\partial x^{2}}(t, x)\right|_{x=0} z^{3}+\left.\frac{1}{4} \frac{\partial^{2}}{\partial x^{2}}\left(h^{2}(t, x)\right)\right|_{x=0} z^{2} .
$$

But

$$
h(t, x)=h(t, 0)\left(1-\eta^{2}\right)^{\frac{1}{3}}, \quad h(0,0)=1,
$$

and therefore

$$
\begin{aligned}
\left.\frac{\partial^{2} h}{\partial x^{2}}(t, x)\right|_{x=0} & =-\frac{2}{3} \frac{h(t, 0)}{w^{2}(t)}, \\
\left.\frac{\partial^{2}}{\partial x^{2}}\left(h^{2}(t, x)\right)\right|_{x=0} & =-\frac{4}{3} \frac{h^{2}(t, 0)}{w^{2}(t)} .
\end{aligned}
$$

Equation (4.6) becomes

$$
v_{z}\left(t, 0, z^{*}\right)=\frac{1}{9} \frac{h^{4}(t, 0)}{w^{2}(t)} z^{* 2}\left(z^{*}-3\right),
$$

where

$$
z^{*}=\frac{z}{h(t, 0)} .
$$

For points in the thin film on the centre line, $0 \leq z^{*} \leq 1$. Now $v_{z}\left(t, 0, z^{*}\right)=0$ at $z^{*}=0$ and $z^{*}=3$ which lies outside the thin film. There is a stagnation point at $x=0, z^{*}=0$ at which $v_{x}=0$ and $v_{z}=0$. There is no stagnation point on the centre line for $0<z^{*} \leq 1$. For $0<z^{*} \leq 1, v_{z}\left(t, 0, z^{*}\right)<0$.

At the point of maximum height of the thin film,

$$
v_{z}(t, 0, h)=-\frac{2}{9} \frac{h^{4}(t, 0)}{w^{2}(t)} .
$$

Using (3.11) and (3.14) for $\beta \neq 1$ we obtain again (4.5). For $\beta=-1$ and using (3.19) and (3.21) we obtain

$$
v_{z}(t, 0, h)=-\frac{2}{9} \exp \left(-\frac{2}{9} t\right) .
$$

The time evolution of $v_{z}(t, 0, h)$ for $-\infty \leq \beta \leq \infty$ is summarised in Table 3.

\section{Streamlines}

The tangent vector to a streamline is everywhere parallel to the fluid velocity vector instantaneously. The streamlines in the $(x, z)$-plane are obtained by solving numerically the cubic Equation (2.30) for $z$,

$$
A(t, x) z^{3}+B(t, x) z^{2}+C(t, x)=K(t)
$$

where the coefficients $A(t, x), B(t, x)$ and $C(t, x)$ are given in (2.21). Now 
from (3.11), (3.14) and (3.19), (3.21),

$$
h(t, x)=w(t)^{-\frac{2}{2+5 \beta}}\left(1-\eta^{2}\right)^{\frac{1}{3}}
$$

and from (3.10), (3.11) and (3.20), (3.21),

$$
v_{n}(t, x)=\frac{10}{27} \beta w(t)^{-2\left(\frac{6+5 \beta}{2+5 \beta}\right)} \frac{\eta^{2}}{\left(1-\eta^{2}\right)^{\frac{2}{3}}} .
$$

Thus from (2.31),

$$
\begin{gathered}
A(t, x)=-\frac{1}{9} w(t)^{-2\left(\frac{3+5 \beta}{2+5 \beta}\right)} \frac{x}{\left(1-\frac{x^{2}}{w^{2}(t)}\right)^{\frac{2}{3}},} \\
B(t, x)=\frac{1}{3} w(t)^{-2\left(\frac{4+5 \beta}{2+5 \beta}\right)} \frac{x}{\left(1-\frac{x^{2}}{w^{2}(t)}\right)^{\frac{1}{3}}}, \\
C(t, x)=-\frac{10}{27} \beta w(t)^{-4\left(\frac{4+5 \beta}{2+5 \beta}\right)} \int_{0}^{x} \frac{s^{2}}{\left(1-\frac{s^{2}}{w^{2}(t)}\right)^{\frac{2}{3}}} \mathrm{~d},
\end{gathered}
$$

where $-w(t) \leq x \leq w(t)$ and $0 \leq z \leq h(t, x)$.

We will mainly consider the streamlines in the $\left(\eta, z^{*}\right)$-plane where $\eta=x / w(t), \quad-1 \leq \eta \leq 1$, and $z^{*}=z / h(t, 0), 0 \leq z^{*} \leq 1$. In this plane the width of the base and the maximum height are constant and the streamline pattern is independent of time and depends only on the parameter $\beta$. The evolution of the streamlines with time can be determined using the evolution with time of the half-width of the base, $w(t)$, and the maximum height $h(t, 0)$ given in Table 2 . In the $\left(\eta, z^{*}\right)$-plane the cubic equation is given by (2.32) with coefficients

$$
\begin{gathered}
A^{*}(t, \eta)=h^{3}(t, 0) A(t, x)=-\frac{1}{9} w(t)^{-5\left(\frac{2+\beta}{2+5 \beta}\right) \frac{\eta}{\left(1-\eta^{2}\right)^{\frac{2}{3}}},} \\
B^{*}(t, \eta)=h^{2}(t, 0) B(t, x)=\frac{1}{3} w(t)^{-5\left(\frac{2+\beta}{2+5 \beta}\right) \frac{\eta}{\left(1-\eta^{2}\right)^{\frac{1}{3}}},} \\
C^{*}(t, \eta)=C(t, x)=-\frac{10}{27} \beta w(t)^{-5}\left(\frac{2+\beta}{2+5 \beta}\right) \int_{0}^{\eta} \frac{\zeta^{2}}{\left(1-\zeta^{2}\right)^{2 / 3}} \mathrm{~d} \zeta .
\end{gathered}
$$

The coefficients $A^{*}(t, \eta), B^{*}(t, \eta)$ and $C^{*}(t, \eta)$ have the same time dependent factor. The cubic Equation (2.32) for $z^{*}$ is

$$
\frac{\eta}{\left(1-\eta^{2}\right)^{2 / 3}} z^{* 3}-3 \frac{\eta}{\left(1-\eta^{2}\right)^{1 / 3}} z^{* 2}+\frac{10}{3} \beta \int_{0}^{\eta} \frac{\zeta^{2}}{\left(1-\zeta^{2}\right)^{2 / 3}} \mathrm{~d} \zeta=K^{*},
$$

where 


$$
K^{*}=-9 w(t)^{5}\left(\frac{2+\beta}{2+5 \beta}\right) K(t) .
$$

Since the left hand side of (5.10) does not depend on time explicitly, $K^{*}$ is a constant. The constant $K^{*}$ is given a range of values. The streamlines in the $\left(\eta, z^{*}\right)$-plane are obtained by solving numerically the cubic Equation (5.10) for $Z^{*}$ with $-1 \leq \eta \leq 1$. The pattern of streamlines is independent of time because (5.10) does not depend on time explicitly. It depends only on the parameter $\beta$.

For $\beta=0$ the base is impermeable and the streamlines in the $\left(\eta, z^{*}\right)$-plane are plotted in [1]. In Figure 2, leak-off is considered and the streamlines are plotted in the $\left(\eta, z^{*}\right)$-plane for strong suction, $\beta=-\frac{1}{2},\left(-\frac{4}{5}<\beta<\frac{2}{5}\right)$ and for weak suction, $\beta=-\frac{1}{5}\left(-\frac{2}{5}<\beta<0\right)$. The streamlines begin on the free surface. They are perpendicular to the base because there is no slip of fluid at the base. The point $(0,0)$ where the centre line meets the base is a stagnation point. Since $v_{n}(t, x)$ is proportional to $x \frac{\partial h}{\partial x}$, near the centre line the suction is weak and spreading due to gravity dominates. As distance from the centre line increases the suction increases and is strongest near the moving contact lines. The streamlines are strongly curved near the centre line because of spreading due to gravity and are weakly curved near the moving contact lines because suction is more important than spreading.

In Figure 3 the streamlines are plotted in the $\left(\eta, z^{*}\right)$-plane for fluid injection (blowing) at the base for $\beta=1,15,30$ and 50. The magnitude of the fluid injection velocity $v_{n}(t, x)$ increases as $\beta$ increases and the blowing becomes stronger. Since $v_{n}(t, x)$ is proportional to $x \frac{\partial h}{\partial x}, v_{n}(t, 0)=0$ and the point $(0,0)$ is again a stagnation point. A dividing streamline extends from this stagnation point and separates the flow into two regions. In the upper region the streamlines begin and end on the free surface. The upper region shows fluid descending due to

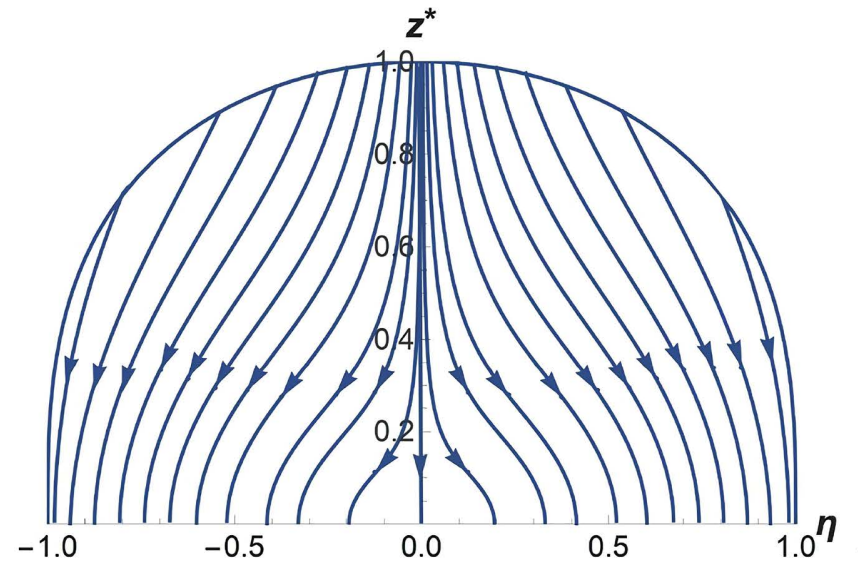

(a)

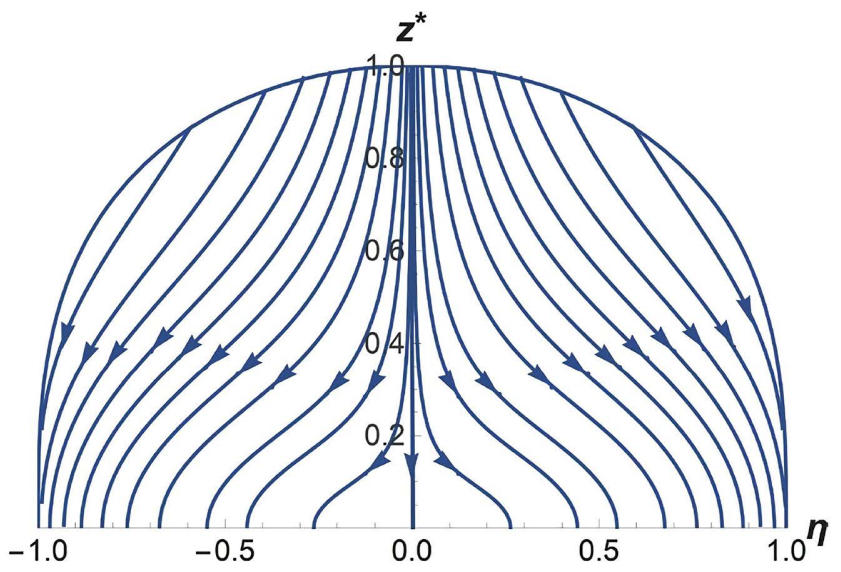

(b)

Figure 2. Streamlines for leak-off at the base with (a) $\beta=-0.5$ and (b) $\beta=-0.2$ plotted in the $\left(\eta, z^{*}\right)$-plane. 


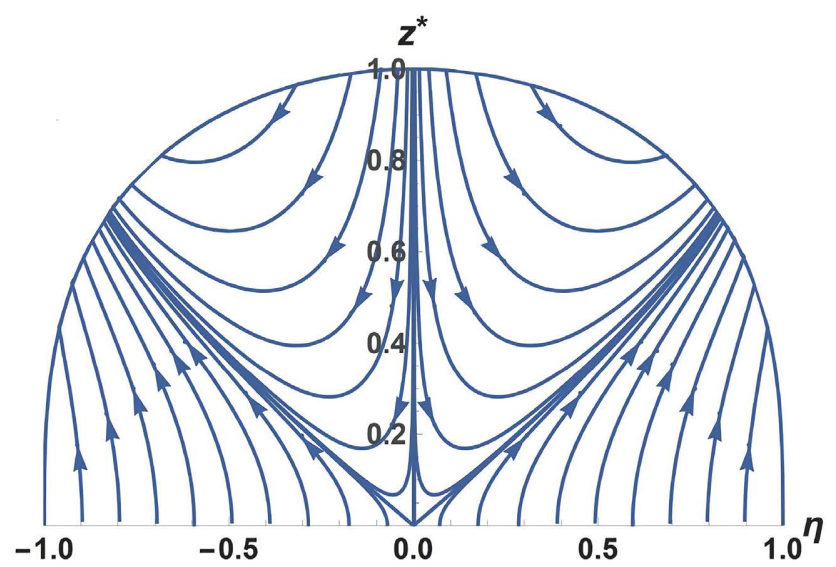

(a)

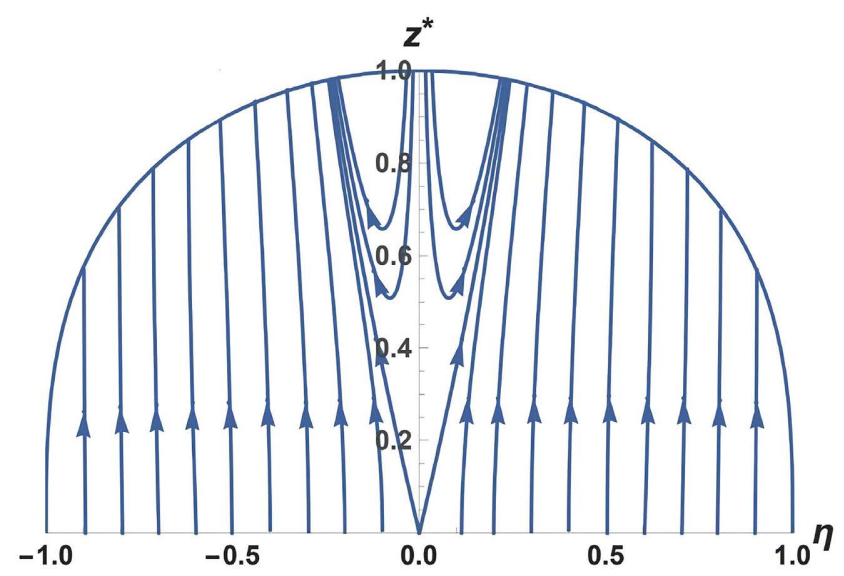

(c)

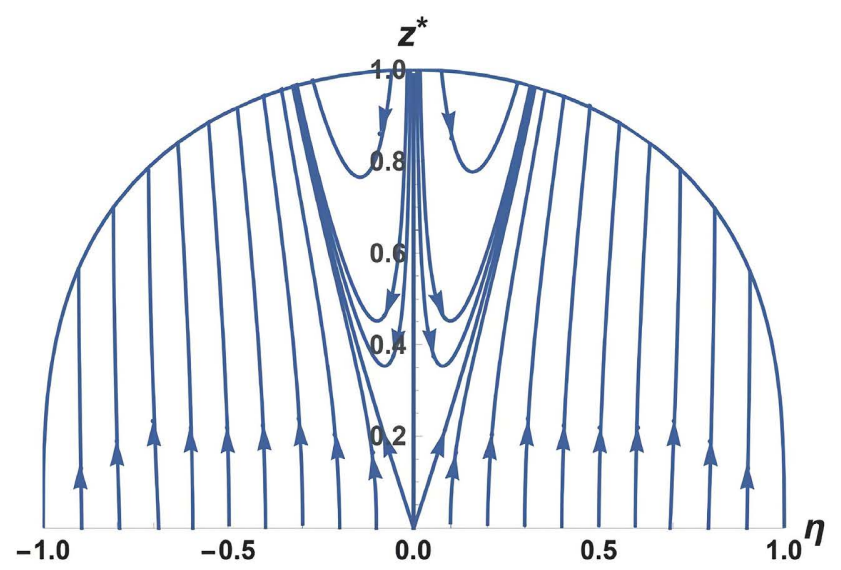

(b)

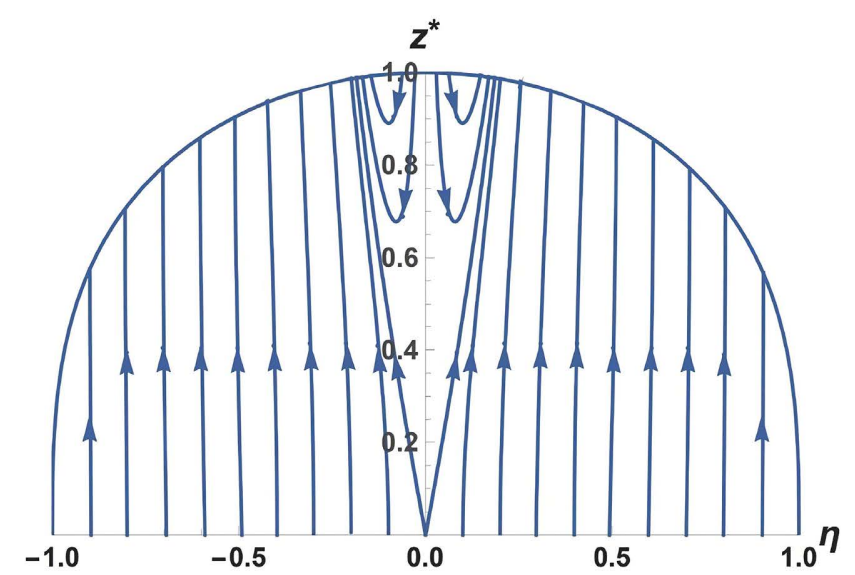

(d)

Figure 3. Streamlines for fluid injection at the base with (a) $\beta=1$, (b) $\beta=15$, (c) $\beta=30$ and (d) $\beta=50$, plotted in the $\left(\eta, z^{*}\right)$-plane.

gravity and spreading. The fluid flow near the centre line is downwards but the flow is forced upwards as it approaches the free surface because of the stronger injection of fluid as the moving contact lines are approached. The lower region describes the injection of fluid into the thin film. The streamlines begin at the base and end on the free surface. The thickness of the fluid layer at the base increases approximately linearly from zero at the stagnation point to the height of the free surface $h(t, x)$ as $|x|$ increases because blowing increases as the moving contact lines are approached. The angle which the dividing streamline makes with the base at the stagnation point increases as $\beta$ increases and therefore as the strength of the blowing increases.

In order to investigate the evolution in time of the streamlines only $w(t)$ and $h(t, 0)$ are required. The evolution of the streamline pattern with time can be determined using

$$
x=w(t) \eta, \quad z=h(t, 0) z^{*}
$$

and the results of Table 2 for the evolution of $w(t)$ and $h(t, 0)$. In Figure 2(a), 
$\beta=-\frac{1}{2}$ which lies in the range of strong suction, $-\frac{4}{5} \leq \beta \leq-\frac{2}{5}$, and $w(t) \rightarrow 0$ and $h(t, 0) \rightarrow 0$ as $t \rightarrow \infty$. The half-width of the base, $w(t)$, and the maximum height, $h(t, 0)$, will decrease steadily with time and the thin fluid film will disappear as a line sink as $t \rightarrow \infty$. In Figure 2(b), $\beta=-\frac{1}{5}$ which lies in the range of weak suction, $-\frac{2}{5}<\beta<0$, and $w(t) \rightarrow \infty$ and $h \rightarrow 0$ as $t \rightarrow \infty$. The half-width of the base, $w(t)$, will increase steadily with time and the maximum height, $h(t, 0)$, will decrease steadily with time. The thin fluid film will spread over the whole plane $z=0$ with decreasing thickness and disappear as $t \rightarrow \infty$. In Figure 3 fluid injection (blowing) is considered and $\beta$ lies in the range $0<\beta<\infty$ for which $w(t) \rightarrow \infty$ and $h(t, 0) \rightarrow 0$ as $t \rightarrow \infty$. The half-with of the base will therefore increase steadily with time and the maximum height of the thin film will decrease steadily with time. The angle which the dividing streamline makes with the base at the stagnation point will therefore decrease steadily with time.

The evolution of the streamline pattern with time is illustrated in Figure 4 where the streamlines are plotted in the $(x, z)$-plane at time $t=1$ and $t=10$. They are obtained by solving numerically the cubic Equation (5.1) for $z$ as a function of $x$ at $t=1$ and $t=10$ where $A(t, x), B(t, x)$ and $C(t, x)$ are given by (5.4) to (5.6).

In Figures $2-4, \beta$ lies in the range $-\frac{4}{5} \leq \beta \leq \infty$ for which the thin fluid film approximation is satisfied for all time. In the next section an approximate analytical solution for the dividing streamline will be derived and its dependence on $\beta$ and its evolution with time will be determined.

\section{Dividing Streamline}

We will derive an approximate solution for the dividing streamline in the $\left(\eta, z^{*}\right)$ -plane. The solution can be expressed in the coordinates $(x, z)$ when required

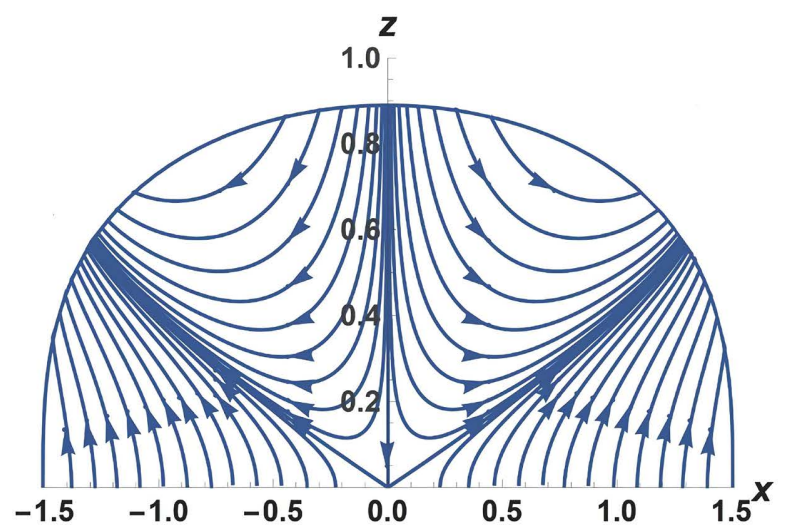

(a)

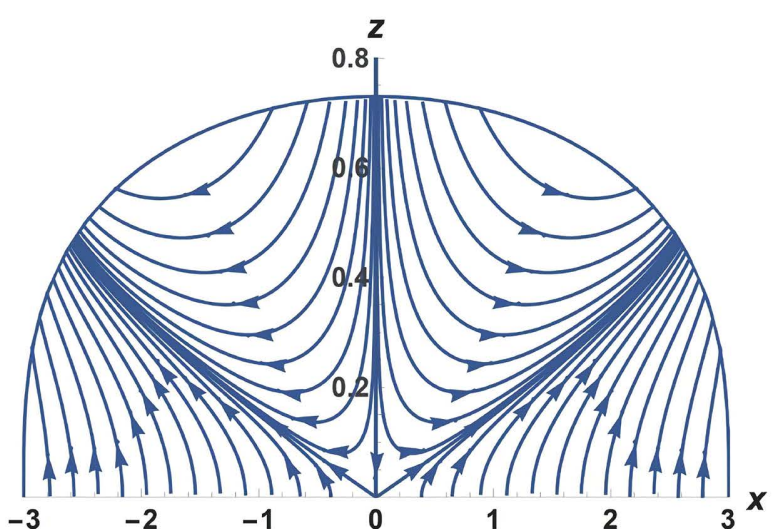

(b)

Figure 4. Streamlines for $\beta=1$ at (a) $t=1$ and (b) $t=10$, plotted in the $(x, z)$-plane. 
later. Since the dividing streamline passes through the stagnation point $(0,0)$, the constant $K^{*}=0$ in (5.10). The cubic Equation (5.10) becomes

$$
z^{* 3}-3\left(1-\eta^{2}\right)^{\frac{2}{3}} z^{* 2}-\frac{10}{3} \beta \frac{\left(1-\eta^{2}\right)^{\frac{2}{3}}}{\eta} \int_{0}^{\eta} \frac{\zeta^{2} \mathrm{~d} \zeta}{\left(1-\zeta^{2}\right)^{2 / 3}}=0 .
$$

In oder to transform (6.1) to standard form [7],

$$
s^{* 3}+3 H s^{*}+G=0 \text {, }
$$

let

$$
z^{*}=s^{*}+\left(1-\eta^{2}\right)^{\frac{1}{3}}
$$

Equation (6.1) becomes

$$
s^{* 3}-3\left(1-\eta^{2}\right)^{\frac{2}{3}} s^{*}-2\left(1-\eta^{2}\right)+\frac{10}{3} \beta \frac{\left(1-\eta^{2}\right)^{\frac{2}{3}}}{\eta} \int_{0}^{\eta} \frac{\zeta^{2} \mathrm{~d} \zeta}{\left(1-\zeta^{2}\right)^{2 / 3}}=0,
$$

with

$$
\begin{gathered}
H(\eta)=-\left(1-\eta^{2}\right)^{\frac{2}{3}} \\
G(\eta)=-2\left(1-\eta^{2}\right)+\frac{10}{3} \beta \frac{\left(1-\eta^{2}\right)^{\frac{2}{3}}}{\eta} \int_{0}^{\eta} \frac{\zeta^{2} \mathrm{~d} \zeta}{\left(1-\zeta^{2}\right)^{2 / 3}} .
\end{gathered}
$$

On the dividing streamline, $0 \leq|\eta|<1$. We perform an expansion in powers of $\eta^{2}$ and derive a solution for blowing, $\beta>0$, to first order in $\eta^{2}$. No assumption is placed on $\beta$ or $t$. We find that this gives a sufficiently accurate result. We see from Figure 3 that as $\beta$ increases the dividing streamline moves closer to the centre line and the maximum value $\zeta^{2}$ on the dividing streamline decreases. The solution should therefore be more accurate as $\beta$ increases.

Now from (6.5) and (6.6),

$$
\begin{gathered}
H(\eta)=-\left(1-\frac{2}{3} \eta^{2}+\mathrm{O}\left(\eta^{4}\right)\right), \\
G(\eta)=-2\left[1-\left(1+\frac{5}{9} \beta\right) \eta^{2}+\mathrm{O}\left(\eta^{4}\right)\right] .
\end{gathered}
$$

The expansion of the cubic equation in powers of $\eta^{2}$ is therefore

$$
s^{* 3}-3\left(1-\frac{2}{3} \eta^{2}+\mathrm{O}\left(\eta^{4}\right)\right) s^{*}-2\left[1-\left(1+\frac{5}{9} \beta\right) \eta^{2}+\mathrm{O}\left(\eta^{4}\right)\right]=0
$$

and from (6.3),

$$
z^{*}=s^{*}+1-\frac{1}{3} \eta^{2}+\mathrm{O}\left(\eta^{4}\right)
$$

The discriminant, $\Delta$, of the cubic Equation (6.9) is [7]

$$
\Delta=G^{2}+4 H^{3}=-\frac{40}{9} \beta \eta^{2}+\mathrm{O}\left(\eta^{4}\right) .
$$


Consider first the solution to zero order in $\eta^{2}$. Then $\Delta=0, H=-1 \neq 0$ and $G=-2 \neq 0$ and therefore the cubic equation has three real roots with two roots coincident and one root different [7]. Equation (6.9) reduces to

$$
\left(s^{*}+1\right)^{2}\left(s^{*}-2\right)=0 \text {. }
$$

Thus $s^{*}=-1, s^{*}=-1, s^{*}=2$ and from (6.10), $z^{*}=0, z^{*}=0$ and $z^{*}=3$. For zero order in $\eta^{2}$ we have $\eta=0$. The point $(0,3)$ lies outside the thin film. The solution for the dividing streamline is the stagnation point $(0,0)$.

For the solution to first order in $\eta^{2}, \Delta<0$ since $\beta>0$ and the cubic equation has three real and distinct roots [7]. We use the trigonometric method of solution described in [1]. The three roots of (6.9) are

$$
s_{n}^{*}=2(-H)^{\frac{1}{2}} \cos \left(\phi+\frac{2 n \pi}{3}\right), \quad n=0,1,2,
$$

where $\phi\left(0 \leq \phi \leq \frac{\pi}{3}\right)$ is the smallest non-negative angle which satisfies

$$
\cos 3 \phi=-\frac{G}{2(-H)^{3 / 2}}=1-\frac{5}{9} \beta \eta^{2}+\mathrm{O}\left(\eta^{4}\right) .
$$

Thus from (6.7) and (6.10),

$$
z_{n}^{*}=\left[1-\frac{1}{3} \eta^{2}+\mathrm{O}\left(\eta^{4}\right)\right]\left[1+\cos \left(\phi+\frac{2 n \pi}{3}\right)\right], \quad n=0,1,2 .
$$

Consider now $\phi$. For $\eta=0, \cos 3 \phi=1$ and therefore $\phi=0$ since $0 \leq \phi \leq \frac{\pi}{3}$. Thus for first order in $\eta^{2}, \phi$ will be small. Using the series expansion for cosine, (6.14) becomes

$$
1-\frac{9}{2} \phi^{2}+\mathrm{O}\left(\phi^{4}\right)=1-\frac{5}{9} \beta \eta^{2}+\mathrm{O}\left(\eta^{4}\right)
$$

and therefore since $0 \leq \phi \leq \frac{\pi}{3}$,

$$
\phi=+\frac{\sqrt{10}}{9} \beta^{\frac{1}{2}} \eta+\mathrm{O}\left(\eta^{3}\right)
$$

Consider first $z_{0}^{*}$ given by (6.15) with $n=0$. Using again the series expansion for cosine and (6.17),

$$
\cos \phi=1-\frac{5}{81} \beta \eta^{2}+\mathrm{O}\left(\eta^{4}\right)
$$

and therefore

$$
z_{0}^{*}=3\left[1-\frac{1}{3}\left(1+\frac{10}{81} \beta\right) \eta^{2}+\mathrm{O}\left(\eta^{4}\right)\right] .
$$

Consider next $z_{1}^{*}$ given by (6.15) with $n=1$. Now

$$
\cos \left(\phi+\frac{2 \pi}{3}\right)=-\frac{1}{2}(\cos \phi+\sqrt{3} \sin \phi) .
$$

But $\cos \phi$ is given by (6.18) and from the series expansion for sine and (6.17) 


$$
\sin \phi=\frac{\sqrt{10}}{9} \beta^{\frac{1}{2}} \eta+\mathrm{O}\left(\eta^{3}\right)
$$

Thus

$$
\cos \left(\phi+\frac{2 \pi}{3}\right)=-\frac{1}{2}\left[1+\frac{\sqrt{30}}{9} \beta^{\frac{1}{2}} \eta-\frac{5}{81} \beta \eta^{2}+\mathrm{O}\left(\eta^{3}\right)\right]
$$

and therefore

$$
z_{1}^{*}=-\frac{\sqrt{30}}{9} \beta^{\frac{1}{2}} \eta\left[1-\frac{\sqrt{30}}{54} \beta^{\frac{1}{2}} \eta+\mathrm{O}\left(\eta^{2}\right)\right] .
$$

Finally, $z_{2}^{*}$ is given by (6.15) with $n=3$. Now

$$
\cos \left(\phi+\frac{4 \pi}{3}\right)=\frac{1}{2}[-\cos \phi+\sqrt{3} \sin \phi]
$$

and using (6.18) and (6.21) we obtain

$$
\cos \left(\phi+\frac{4 \pi}{3}\right)=\frac{1}{2}\left[-1+\frac{\sqrt{30}}{9} \beta^{\frac{1}{2}} \eta+\frac{5}{81} \beta \eta^{2}+\mathrm{O}\left(\eta^{3}\right)\right] .
$$

Thus

$$
z_{2}^{*}=\frac{\sqrt{30}}{9} \beta^{\frac{1}{2}} \eta\left[1+\frac{\sqrt{30}}{54} \beta^{\frac{1}{2}} \eta+\mathrm{O}\left(\eta^{2}\right)\right] .
$$

The dividing streamline must pass through the stagnation point $(0,0)$ and satisfy $z^{*} \geq 0$. The root $z^{*}=z_{0}^{*}(\eta)$ is not a dividing streamline because it does not pass through $(0,0)$. The dividing streamline in the $\left(\eta, z^{*}\right)$ plane correct to order $\eta^{2}$ is

$$
\begin{aligned}
0 \leq \eta \leq 1: \quad z^{*}=z_{2}^{*}(\eta) & =\frac{\sqrt{30}}{9} \beta^{\frac{1}{2}} \eta\left[1+\frac{\sqrt{30}}{54} \beta^{\frac{1}{2}} \eta+\mathrm{O}\left(\eta^{2}\right)\right], \\
-1 & \leq \eta \leq 0: \quad z^{*}=z_{1}^{*}(\eta)=-\frac{\sqrt{30}}{9} \beta^{\frac{1}{2}} \eta\left[1-\frac{\sqrt{30}}{54} \beta^{\frac{1}{2}} \eta+\mathrm{O}\left(\eta^{2}\right)\right] .
\end{aligned}
$$

Let $\theta(\beta)$ be the angle that the dividing streamline makes with the positive $\eta$ -axis at the stagnation point $(0,0)$. Then

$$
\tan \theta(\beta)=\left.\frac{\partial z_{2}^{*}}{\partial \eta}\right|_{\eta=0}=\frac{\sqrt{30}}{9} \beta^{\frac{1}{2}} .
$$

Thus $\theta(\beta)$ increases as $\beta$ increases. In Figure 3 the streamlines are plotted in the $\left(\eta, z^{*}\right)$ plane for $\beta=1,15,30$ and 50. From (6.29) the corresponding values of $\theta(\beta)$ are $\theta(1)=31.3^{\circ}, \theta(15)=67.0^{\circ}, \theta(30)=73.3^{\circ}$ and $\theta(50)=76.9^{\circ}$, which agree well with the slope of the dividing streamlines at $(0$, 0 ) in Figure 3.

Let the point of intersection, $P$, of the dividing streamline with the surface of the thin film in the $\left(\eta, z^{*}\right)$-plane be $\left(\eta_{P}, z_{P}^{*}\right)$. Then from (3.14),

$$
z_{P}^{*}=\left(1-\eta_{P}^{2}\right)^{\frac{1}{3}}=1-\frac{1}{3} \eta_{P}^{2}+\mathrm{O}\left(\eta_{P}^{4}\right)
$$


and therefore from (6.27) for $\eta_{P}>0$,

$$
1-\frac{1}{3} \eta_{P}^{2}+\mathrm{O}\left(\eta_{P}^{4}\right)=\frac{\sqrt{30}}{9} \beta^{\frac{1}{2}} \eta_{P}\left[1+\frac{\sqrt{30}}{54} \beta^{\frac{1}{2}} \eta_{P}+\mathrm{O}\left(\eta_{P}^{2}\right)\right] .
$$

Thus neglecting terms $\mathrm{O}\left(\eta_{P}^{3}\right), \eta_{P}$ satisfies the quadratic equation

$$
\left(1+\frac{5}{27} \beta\right) \eta_{P}^{2}+\frac{\sqrt{30}}{3} \beta^{\frac{1}{2}} \eta_{P}-3=0
$$

If terms of order $\eta_{P}^{2}$ are neglected then the point $P$ is

$$
\eta_{P}=\frac{9}{\sqrt{30}} \frac{1}{\beta^{1 / 2}}, \quad z_{P}^{*}=1
$$

This approximation for $\eta_{P}$ and $z_{P}^{*}$ is consistent with (6.29) for $\tan \theta(\beta)$ because approximately

$$
\tan \theta(\beta)=\frac{z_{P}^{*}}{\eta_{P}}=\frac{\sqrt{30}}{9} \beta^{\frac{1}{2}}
$$

As $\beta$ increases and the strength of the blowing increases, $\eta_{p}$ decreases, verifying that the dividing streamline moves closer to the centre line. The product $\beta^{\frac{1}{2}} \eta_{P}$ occurs in the expansions (6.27) and (6.28) which are valid because $\eta_{P} \beta^{\frac{1}{2}}$ is of order of magnitude unity. The approximation correct to order $\eta^{2}$ is obtained by solving the quadratic Equation (6.32). The positive root of (6.32) can be expressed as

$$
\eta_{P}=\frac{\sqrt{3}}{1+\frac{5}{27} \beta}\left[\left(1+\frac{25}{54} \beta\right)^{\frac{1}{2}}-\frac{\sqrt{10}}{6} \beta^{\frac{1}{2}}\right] .
$$

The evolution with time can again be determined by using the transformation (5.12). Let $\theta(t)$ be the angle which the dividing streamline in the $(x, z)$-plane makes with the positive $x$-axis at the stagnation point. Then

$$
\tan \theta(t)=\left.\frac{\partial z}{\partial x}\right|_{x=0}=\left.\frac{h(t, 0)}{w(t)} \frac{\partial z^{*}}{\partial \eta}\right|_{\eta=0}
$$

and using (6.29) with (3.11) and (3.14) at $\eta=0$ for $w(t)$ and $h(t, 0)$ we obtain

$$
\tan \theta(t)=\frac{\sqrt{30}}{9} \beta^{\frac{1}{2}}\left[1+\frac{10}{9}(1+\beta) t\right]^{-\left(\frac{4+5 \beta}{10(1+\beta)}\right)} .
$$

The asymptotic solution for large values of $\beta$ is

$$
\tan \theta(t)=\frac{\sqrt{30}}{9} \beta^{\frac{1}{2}}\left(\frac{10}{9} \beta t\right)^{-\frac{1}{2}}=\frac{1}{\sqrt{3} t^{\frac{1}{2}}} .
$$

Thus $\tan \theta(t)$ decreases as time increases. For large values of $\beta$ corresponding to strong blowing the gradient of the dividing streamline at the stagnation point decreases with time like $t^{-\frac{1}{2}}$ and is independent of $\beta$. This makes quantitative 
the conclusion reached in Section 5 on the evolution of $\theta(t)$ with time.

Let $\left(x_{P}, z_{P}\right)$ be the point of intersection of the dividing streamline with the surface of the thin film in the $(x, z)$-plane. Then

$$
x_{P}=w(t) \eta_{P}, \quad z_{P}=h(t, 0) z_{P}^{*},
$$

where $w(t)$ and $h(t, 0)$ are given by (3.11) and by (3.14) at $\eta=0$. Neglecting terms of order $\eta_{P}^{2}$ and using (6.33),

$$
x_{P}=\frac{9}{\sqrt{30} \beta^{\frac{1}{2}}}\left[1+\frac{10}{9}(1+\beta) t\right]^{\frac{2+5 \beta}{10(1+\beta)}}, \quad z_{P}=\left[1+\frac{10}{9}(1+\beta) t\right]^{-\frac{1}{5(1+\beta)}}
$$

and therefore for large $\beta$,

$$
x_{P}=\sqrt{3} t^{\frac{1}{2}}, \quad Z_{P}=1 .
$$

This approximation for $x_{p}$ and $z_{p}$ is consistent with (6.38) for $\tan \theta(t)$ because approximately

$$
\tan \theta(t)=\frac{z_{P}}{x_{P}}=\frac{1}{\sqrt{3} t^{\frac{1}{2}}} .
$$

Keeping terms of order $\eta_{P}^{2}$ we obtain from (6.30),

$$
x_{P}=w(t) \eta_{P}, \quad z_{P}=\left[1-\frac{1}{3} \frac{x_{P}^{2}}{w(t)^{2}}\right] w(t)^{-\frac{2}{2+5 \beta}},
$$

where $\eta_{P}$ is given by (6.35) and

$$
h(t, 0)=w(t)^{-\frac{2}{2+5 \beta}} .
$$

In the $(x, z)$-plane the dividing streamline is obtained by solving numerically for $z$ the cubic Equation (5.1) with $K(t)=0$ and $A(t, x), B(t, x)$ and $C(t, x)$ given by (5.4) to (5.6). From (6.27) and (6.28) the approximate solution is, correct to second order in $x / w(t)$,

$$
\begin{gathered}
0 \leq x \leq w(t): z=\frac{\sqrt{30}}{9} \beta^{\frac{1}{2}} \frac{x}{w(t)}\left[1+\frac{\sqrt{30}}{54} \beta^{\frac{1}{2}} \frac{x}{w(t)}\right] w(t)^{-\frac{2}{2+5 \beta}}, \\
-w(t) \leq x \leq 0: z=-\frac{\sqrt{30}}{9} \beta^{\frac{1}{2}} \frac{x}{w(t)}\left[1-\frac{\sqrt{30}}{54} \beta^{\frac{1}{2}} \frac{x}{w(t)}\right] w(t)^{-\frac{2}{2+5 \beta}} .
\end{gathered}
$$

In Figure 5 the approximate solution, (6.45) and (6.46), for the dividing streamline is plotted in the $(x, z)$ plane for $\beta=50$ and times ranging from $t=5$ to $t=1000$. We see clearly that as $t$ increases the angle $\theta(t)$ that the dividing streamline makes with the $x$-axis steadily decreases because spreading is stronger than blowing near the centre line. In Figure 6 the approximate solution, (6.45) and (6.46), for the dividing streamline is compared with the numerical solution of (5.1) with $K(t)=0$. The approximate solution is more accurate as $\beta$ increases. The approximate solution (6.43) for $\left(x_{p}, z_{P}\right)$ is also compared with the numerical solution and its accuracy also increases as $\beta$ increases. Figure 6 also 


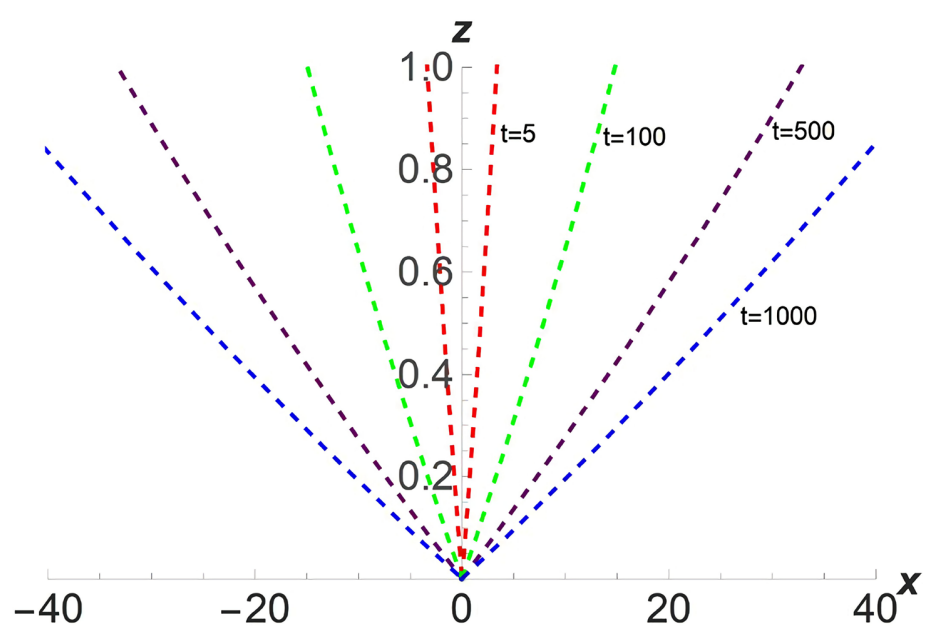

Figure 5. Approximate solution for the dividing streamline in the $(x, z)$-plane given by (6.45) and (6.46) for $\beta=50$ at time $t=5, t=100, t=500$ and $t=1000$.

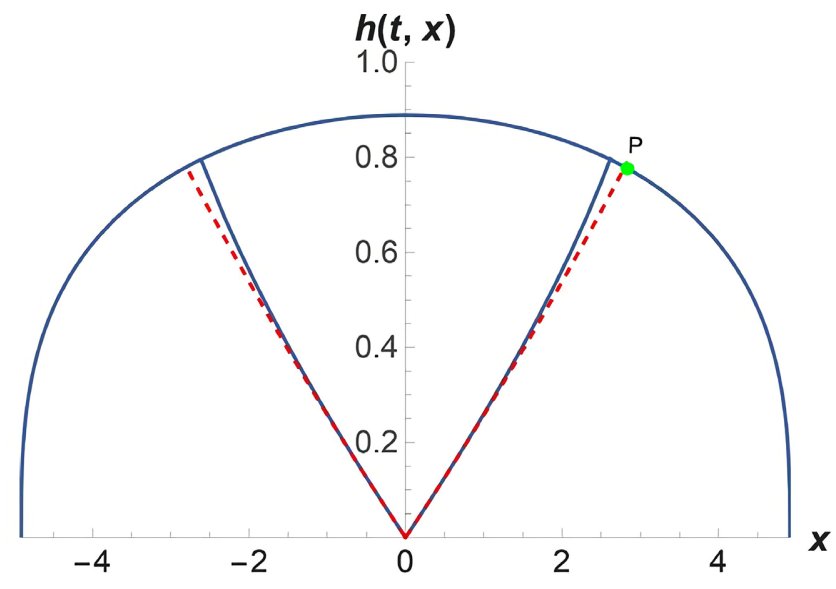

(a)

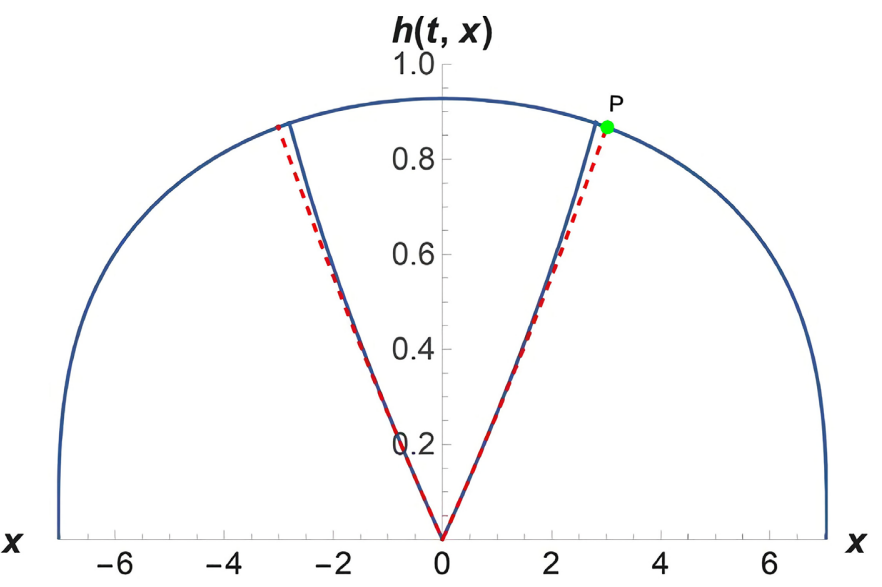

(b)

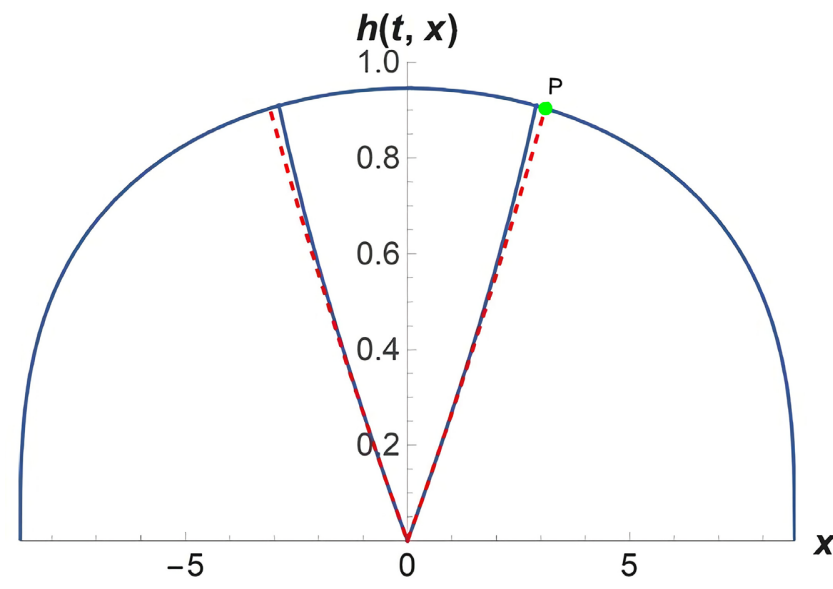

(c)

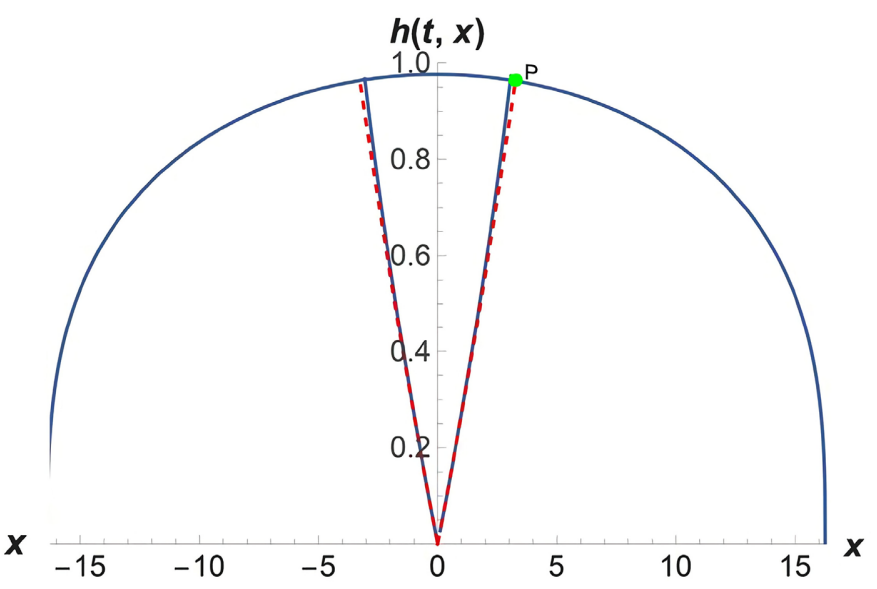

(d)

Figure 6. Approximate solution for the dividing streamline in the $(x, z)$-plane given by (6.45) and (6.46) (----) compared with the numerical solution (-) at time $t=5$ and (a) $\beta=5$, (b) $\beta=100$, (c) $\beta=500$ and (d) $\beta=1000$. The point of intersection, $P$, of the dividing streamline with the surface is given approximately by (6.43). 
illustrates the increase in the base, $2 w(t)$, with time and the decrease during blowing of the maximum height although the rate of decrease of $h(t, 0)$ decreases as $\beta$ increases. From (3.14) for large values of $\beta$,

$$
\frac{\mathrm{d} h}{\mathrm{~d} t}(t, 0) \sim-\frac{1}{5 \beta t} \text {. }
$$

\section{Conclusions}

Analysis of the streamlines inside the thin fluid film contributed to the understanding of the effect suction and blowing at the base has on the fluid flow inside the thin film. The properties could be analysed both analytically and numerically because an invariant solution could be derived when $v_{n}$ is proportional to $x \frac{\partial h}{\partial x}$.

The properties of the fluid flow in this study and in [1] where $v_{n}$ is proportional to $h(t, x)$ can be expected to be present in other models for suction and blowing. If more realistic models cannot be solved analytically these properties will be a useful guide in a numerical solution. In both studies, there is a range of suction and blowing for which the thin fluid film approximation is satisfied and outside this range, the solutions are not physically acceptable. In both models a dividing streamline exists, starting at a stagnation point, which separates the flow into two regions, a lower region at the base consisting of rising fluid and an upper region consisting mainly of descending fluid. Both models have fluid variables which tend to zero or infinity in a finite time. The transition solution to an infinite limiting time is an exponential solution.

For the study presented here the thin fluid film approximation was satisfied for $-\frac{4}{5} \leq \beta \leq \infty$, that is, for moderate to weak suction and the whole range of blowing. The exponential solution, generated by a special case of the Lie point symmetry, and the solutions which tended to zero or infinity in a finite time, lie outside this range. The study complemented the investigation in [1] for which the thin fluid film approximation was satisfied for $-\infty<\beta \leq 2$, that is, for the whole of suction and for weak to moderate blowing. There was unexpected behaviour in the evolution of $w(t)$ and $h(t, 0)$ caused by the relative importance of spreading due to gravity and suction or blowing. The base half-width $w(t) \rightarrow \infty$ for all blowing and even for weak suction for which $-\frac{2}{5}<\beta<0$. The maximum height $h(t, 0) \rightarrow 0$ for all suction and also for all blowing for which $0<\beta<\infty$, although $\frac{\mathrm{d} h}{\mathrm{~d} t}(t, 0)$ decreases as $\beta$ increases. Because blowing is weakest at the centre line and strongest at the moving contact lines the dividing streamline generated an approximate $\mathrm{V}$-shaped surface along the length of the two-dimensional thin fluid film, with vertex of each section at a stagnation point. This compares with the dividing streamline in [1] which also passed through a stagnation point and generated an approximately horizontal surface that dipped down at the moving contact lines. 
Further research can be undertaken. Surface tension was neglected and there was no slip at the fluid/base interface. The streamlines in a thin fluid film with surface tension or slip could be investigated to give a better understanding of the fluid film flow inside the thin film.

\section{Acknowledgements}

DPM thanks the National Research Foundation, Pretoria, South Africa, for financial support under grant number 132189.

\section{Conflicts of Interest}

The authors declare no conflicts of interest regarding the publication of this paper.

\section{References}

[1] Modhien, N., Mason, D.P. and Momoniat, E. (2021) Streamlines in the Two-Dimensional Spreading of a Thin Fluid Film: Blowing and Suction Velocity Proportional to the Height. Journal of Applied Mathematics and Physics, 9, 2114-2151. https://doi.org/10.4236/jamp.2021.98133

[2] Momoniat, E. and Abelman, S. (2004) An Investigation into the Spreading of a Thin Liquid Drop under Gravity on a Slowly Rotating Disk. International Journal of Non-Linear Mechanics, 39, 265-270. https://doi.org/10.1016/S0020-7462(02)00173-7

[3] Momoniat, E., Mason, D.P. and Mahomed, F.M. (2001) Non-Linear Diffusion of an Axisymmetric Liquid Drop: Group Invariant Solution and Conservation Law. International Journal of Non-Linear Mechanics, 36, 879-885. https://doi.org/10.1016/S0020-7462(00)00051-2

[4] Momoniat, E., Myers, T.G. and Abelman, S. (2005) New Solutions for Surface Tension Driven Spreading of a Thin Film. Inter. International Journal of Non-Linear Mechanics, 40, 523-529. https://doi.org/10.1016/j.ijnonlinmec.2004.07.017

[5] Mason, D.P. and Momoniat, E. (2004) Axisymmetric Spreading of a Thin Liquid Drop with Suction or Blowing at the Horizontal Base. International Journal of NonLinear Mechanics, 39, 1013-1026. https://doi.org/10.1016/S0020-7462(03)00093-3

[6] Ibragimov, N.H. and Anderson, R.L. (1994) Apparatus of Group Analysis. In: Ibragimov, N.H., Ed., CRC Handbook of Lie Group Analysis of Differential Equations, Vol. 1, Symmetries, Exact Solutions and Conservation Laws, CRC Press, Boca Raton, 12-14.

[7] Barnard, S. and Child, J.M. (1936) Higher Algebra. MacMillan, London. 